Communications in Physics, Vol.30, No. 1 (2020), pp. 35-47

DOI:10.15625/0868-3166/30/1/14461

\title{
POLARIZATION OBSERVABLES IN WZ PRODUCTION AT THE 13 TEV LHC: INCLUSIVE CASE
}

\author{
JULIEN BAGLIO $^{1}$ AND LE DUC NINH ${ }^{2, \dagger}$ \\ ${ }^{1}$ CERN, Theoretical Physics Department, CH-1211 Geneva 23, Switzerland \\ ${ }^{2}$ Institute For Interdisciplinary Research in Science and Education, \\ ICISE, 590000 Quy Nhon, Vietnam \\ ${ }^{\dagger} E$-mail: 1dninh@ifirse.icise.vn \\ Received 4 November 2019
}

Accepted for publication 21 January 2020

Published 28 February 2020

\begin{abstract}
We present a study of the polarization observables of the $W$ and $Z$ bosons in the process $p p \rightarrow W^{ \pm} Z \rightarrow e^{ \pm} v_{e} \mu^{+} \mu^{-}$at the $13 \mathrm{TeV}$ Large Hadron Collider. The calculation is performed at next-to-leading order, including the full QCD corrections as well as the electroweak corrections, the latter being calculated in the double-pole approximation. The results are presented in the helicity coordinate system adopted by ATLAS and for different inclusive cuts on the di-muon invariant mass. We define left-right charge asymmetries related to the polarization fractions between the $W^{+} Z$ and $W^{-} Z$ channels and we find that these asymmetries are large and sensitive to higher-order effects. Similar findings are also presented for charge asymmetries related to a $P$-even angular coefficient.
\end{abstract}

Keywords: diboson production, LHC, next-to-leading-order corrections, polarization, standard model.

Classification numbers: 12.15.-y; 14.70.Fm 14.70.Hp.

\section{INTRODUCTION}

In the framework of the Standard Model (SM) of particle physics the $W$ boson only interacts with left-handed fermions while the $Z$ boson interacts with both left- and right-handed fermions, albeit with different coupling strengths. This allows for a polarized production at hadron colliders and in particular at the CERN Large Hadron Collider (LHC), leading to asymmetries in the angular distributions of the leptonic decay products of the electroweak gauge bosons. Measuring these 
asymmetries is a probe of the underlying polarization of the gauge bosons and eventually of their spin structure.

The pair production of $W$ and $Z$ bosons has been the subject of recent experimental studies [1] in order to gain information about the polarization of the gauge bosons. On the theory side, leading order (LO) studies began a while ago [2,3] before being revived [4] and studied in a recent paper [5] in the process $p p \rightarrow W^{ \pm} Z \rightarrow e^{ \pm} v_{e} \mu^{+} \mu^{-}$at next-to-leading order (NLO) including both QCD and electroweak (EW) corrections, the latter being calculated in the doublepole approximation (DPA). This approximation works remarkably well in this process as shown by the comparison performed in Ref. [5] with the exact NLO EW calculation for the differential distributions [6], which completed the NLO EW picture after the on-shell predictions presented in Refs. $[7,8]$. Note that for the production process itself the QCD corrections are known up to nextto-next-to-leading order in QCD [9-12]. In Ref. [5] the extensive study of the NLO QCD+EW predictions for gauge boson polarization observables, namely polarization fractions and angular coefficients, was done in two different coordinate systems, the Collins-Soper [13] and helicity [14] coordinate systems. However, in the recent experimental analysis by ATLAS with $13 \mathrm{TeV}$ LHC data [1], a different coordinate system was used, namely a modified helicity coordinate system in which the $z$ axis is now defined as the direction of the $W$ (or $Z$ ) boson as seen in the $W Z$ center-ofmass system. In addition, the study in Ref. [5] introduced fiducial polarization observables, which have the advantage of being much simpler to define and calculate (and should also be measurable), but are not the observables that are measured by the experiments yet.

The goal of this paper is to make one step closer to the experimental setup by using the modified helicity coordinate system and giving predictions in an inclusive setup, using as default the experimental total phase space defined by ATLAS at the $13 \mathrm{TeV}$ LHC. It is noted that polarization fractions at the total-phase-space level are needed in [1] to simulate the helicity templates necessary to extract the polarization fractions in the fiducial-phase-space region.

In addition to providing results for polarization fractions and angular coefficients, we also present two charge asymmetries (denoted $\mathscr{A}_{L R}^{V}$ and $\mathscr{C}_{3}^{V}$ with $V=W, Z$ ) that are large at the NLO QCD+EW accuracy, which are sensitive to either the QCD or the EW corrections depending on the asymmetry and on the gauge boson that is under consideration. These asymmetries help to probe the underlying spin structure of the gauge bosons and should be measurable in the experiments. We use the same calculation setup presented in Ref. [5], and our calculation is exact at NLO QCD using the program VBFNLO $[15,16]$ while the EW corrections are calculated in the DPA presented in Ref. [5].

Compared to our previous work [5], the differences in this paper are the phase-space cuts and the definition of the coordinate system to determine the lepton angles. Instead of using more realistic fiducial cuts as in Ref. [5], we use here only one simple cut on the muon-pair invariant mass, e.g. $66 \mathrm{GeV}<m_{\mu^{+} \mu^{-}}<116 \mathrm{GeV}$. The benefit of considering this inclusive phase space is that the "genuine" polarization observables can be easily calculated using the projection method defined in Ref. [5]. The word "genuine" here means that the observables are not affected by cuts on the kinematics of the individual leptons such as $p_{T, \ell}$ or $\eta_{\ell}$. This inclusive case may therefore provide more insights into various effects, which are difficult to understand when complicated kinematical cuts on the individual leptons are present.

We discuss in Sec. II the polarization observables of a massive gauge boson in the total phase space and our method to calculate them. The coordinate system that we use to determine 
the lepton angles is also defined. In Sec. III numerical results for the polarization fractions and angular coefficients are presented for both $W^{+} Z$ and $W^{-} Z$ channels. From this, results for various charge asymmetries between the two channels are calculated. We finally conclude in Sec. IV.

\section{POLARIZATION OBSERVABLES}

The definition of polarization observables and calculational details have been all given in Ref. [5] and we will not repeat them extensively. For an easy reading of this paper, we provide here a brief summary of polarization observables and the main calculational details. This will be needed to understand the numerical results presented in the next section. Polarization observables associated with a massive gauge boson are constructed based on the angular distribution of its decay product, typically a charged lepton (electron or muon). In the rest frame of the gauge boson, this distribution reads $[13,17,18]$

$$
\begin{aligned}
\frac{d \sigma}{\sigma d \cos \theta d \phi} & =\frac{3}{16 \pi}\left[\left(1+\cos ^{2} \theta\right)+A_{0} \frac{1}{2}\left(1-3 \cos ^{2} \theta\right)+A_{1} \sin (2 \theta) \cos \phi\right. \\
& +A_{2} \frac{1}{2} \sin ^{2} \theta \cos (2 \phi)+A_{3} \sin \theta \cos \phi+A_{4} \cos \theta \\
& \left.+A_{5} \sin ^{2} \theta \sin (2 \phi)+A_{6} \sin (2 \theta) \sin \phi+A_{7} \sin \theta \sin \phi\right],
\end{aligned}
$$

where $\theta$ and $\phi$ are the lepton polar and azimuthal angles, respectively, in a particular coordinate system that needs to be specified. $A_{0-7}$ are dimensionless angular coefficients independent of $\theta$ and $\phi . A_{0-4}$ are called P-even and $A_{5-7}$ P-odd according to the parity transformation where $\phi$ flips sign while $\theta$ remains unchanged $[19,20]$. We also note here that $A_{5-7}$ are proportional to the imaginary parts of the spin-density matrix of the $W$ and $Z$ bosons in the DPA at LO [5, 21,22]. This is important to understand why the values of these coefficients are very small, as will be later shown.

We can also define the polarization fractions $f^{W / Z}$ by integrating over $\phi$,

$$
\begin{aligned}
& \frac{d \sigma}{\sigma d \cos \theta_{e^{ \pm}}}=\frac{3}{8}\left[\left(1 \mp \cos \theta_{e^{ \pm}}\right)^{2} f_{L}^{W^{ \pm}}+\left(1 \pm \cos \theta_{e^{ \pm}}\right)^{2} f_{R}^{W^{ \pm}}+2 \sin ^{2} \theta_{e^{ \pm}} f_{0}^{W^{ \pm}}\right], \\
& \frac{d \sigma}{\sigma d \cos \theta_{\mu^{-}}}=\frac{3}{8}\left[\left(1+\cos ^{2} \theta_{\mu^{-}}+2 c \cos \theta_{\mu^{-}}\right) f_{L}^{Z}+\left(1+\cos ^{2} \theta_{\mu^{-}}-2 c \cos \theta_{\mu^{-}}\right) f_{R}^{Z}+2 \sin ^{2} \theta_{\mu^{-}} f_{0}^{Z}\right] .
\end{aligned}
$$

The upper signs are for $W^{+}$and the lower signs are for $W^{-}$. The parameter $c$ reads

$$
c=\frac{g_{L}^{2}-g_{R}^{2}}{g_{L}^{2}+g_{R}^{2}}=\frac{1-4 s_{W}^{2}}{1-4 s_{W}^{2}+8 s_{W}^{4}}, \quad s_{W}^{2}=1-\frac{M_{W}^{2}}{M_{Z}^{2}},
$$

occurring because the $Z$ boson decays into both left- and right-handed leptons. Relations between the polarization fractions $f_{L, R, 0}^{V}$ with $V=W, Z$ and the angular coefficients are therefore obvious,

$$
f_{L}^{V}=\frac{1}{4}\left(2-A_{0}^{V}+b_{V} A_{4}^{V}\right), f_{R}^{V}=\frac{1}{4}\left(2-A_{0}^{V}-b_{V} A_{4}^{V}\right), f_{0}^{V}=\frac{1}{2} A_{0}^{V}
$$

where $b_{W^{ \pm}}=\mp 1, b_{Z}=1 / c$. From this, we get

$$
f_{L}^{V}+f_{R}^{V}+f_{0}^{V}=1, \quad f_{L}^{V}-f_{R}^{V}=\frac{b_{V}}{2} A_{4}^{V}
$$


These coefficients are named polarization observables because they are directly related to the spin-density matrix of the $W$ and $Z$ bosons in the DPA and at LO as above mentioned. In order to calculate them, we first have to calculate the distributions $d \sigma /(d \cos \theta d \phi)$, or simply the distributions $d \sigma /(d \cos \theta)$ if only the polarization fractions are of interest. This can be computed order by order in perturbation theory. We have calculated this up to the NLO QCD + EW accuracy using the same calculation setup as in Ref. [5]. The NLO QCD results are exact, using the full amplitudes as provided by the VBFNLO program. The NLO EW corrections are however calculated in the DPA as presented in Ref. [5]. In the DPA, only the double-resonant Feynman diagrams are taken into account. Single-resonant diagrams including $\gamma^{*} \rightarrow \mu^{+} \mu^{-}$(as shown in Fig. 1a) or $W \rightarrow 2 \ell 2 v$ (as shown in Fig. 1b) are neglected. Moreover, even for the double-resonant diagrams, off-shell effects are not included. In the next section we will also provide results at LO using the DPA (dubbed DPA LO) or using the full amplitudes (dubbed simply LO).
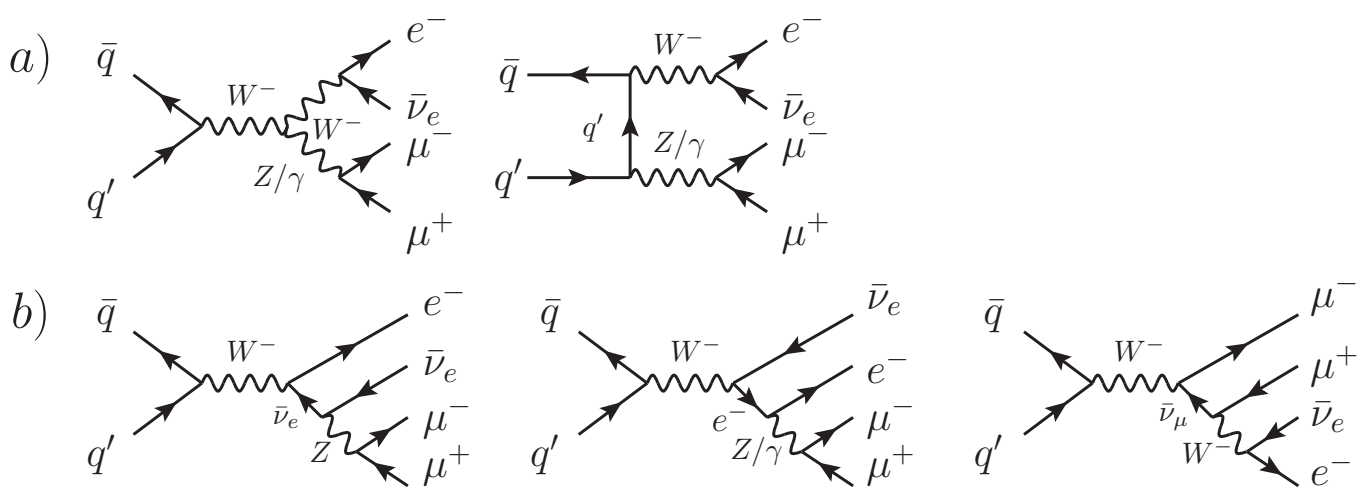

Fig. 1. Double and single resonant diagrams at leading order. Group a) includes both double and single resonant diagrams, while group b) is only single resonant.

Finally, we specify the coordinate system to determine the angle $\theta$ and $\phi$. Differently from Ref. [5], we use here the modified helicity coordinate system. The only difference compared to the helicity system is the direction of the $z$ axis: instead of being the gauge boson flight direction in the laboratory frame as chosen in Refs. [5,14], it is now the gauge boson flight direction in the $W Z$ center-of-mass frame. This modified helicity coordinate system is also used in the latest ATLAS paper presenting results for the polarization observables in the $W Z$ channel [1]. We think the modified helicity system is a better choice when studying the spin correlations of the two gauge bosons. However, for polarizations of a single gauge boson, the helicity system is more advantageous because of a better reconstruction of the $Z$ boson direction in the laboratory frame. In both cases, an algorithm to determine the momentum of the $W$ boson from its decay products is still needed, which has been done in [1]. We note that the spin correlations of the two gauge bosons are fully included in our calculation. However, we do not provide separately numerical results for these effects in this paper because the need for them is not urgent as the current experimentalstatistic level is still limited to be sensitive to those effects. Nevertheless, we choose to use the modified helicity system to be closer to the ATLAS measurement and to prepare for the future studies of those spin correlations. 


\section{NUMERICAL RESULTS}

The input parameters are

$$
\begin{array}{r}
G_{\mu}=1.16637 \times 10^{-5} \mathrm{GeV}^{-2}, M_{W}=80.385 \mathrm{GeV}, M_{Z}=91.1876 \mathrm{GeV}, \\
\Gamma_{W}=2.085 \mathrm{GeV}, \Gamma_{Z}=2.4952 \mathrm{GeV}, M_{t}=173 \mathrm{GeV}, M_{H}=125 \mathrm{GeV},
\end{array}
$$

which are the same as the ones used in Ref. [5]. The masses of the leptons and the light quarks, i.e. all but the top mass, are approximated as zero. This is justified because our results are insensitive to those small masses. The electromagnetic coupling is calculated as $\alpha_{G_{\mu}}=\sqrt{2} G_{\mu} M_{W}^{2}(1-$ $\left.M_{W}^{2} / M_{Z}^{2}\right) / \pi$. For the factorization and renormalization scales, we use $\mu_{F}=\mu_{R}=\left(M_{W}+M_{Z}\right) / 2$. Moreover, the parton distribution functions (PDF) are calculated using the Hessian set LUXqed17_plus_PDF4LHC15_nnlo_30 [23-32] via the library LHAPDF6 [33].

We will give results for the LHC running at a center-of-mass energy $\sqrt{s}=13 \mathrm{TeV}$, for both $e^{+} v_{e} \mu^{+} \mu^{-}$and $e^{-} \bar{v}_{e} \mu^{+} \mu^{-}$final states, also denoted, respectively, as $W^{+} Z$ and $W^{-} Z$ channels for conciseness. We treat the extra parton occurring in the NLO QCD corrections inclusively and we do not apply any jet cuts. We also consider the possibility of lepton-photon recombination, where we redefine the momentum of a given charged lepton $\ell$ as being $p_{\ell}^{\prime}=p_{\ell}+p_{\gamma}$ if $\Delta R(\ell, \gamma) \equiv$ $\sqrt{(\Delta \eta)^{2}+(\Delta \phi)^{2}}<0.1$. We use $\ell$ for either $e$ or $\mu$. If not otherwise stated, the default phase-space cut is

$$
66 \mathrm{GeV}<m_{\mu^{+} \mu^{-}}<116 \mathrm{GeV},
$$

which is used in Ref. $[1,34]$ to define the experimental total phase space. With this cut, we obtain the following result for the total cross section

$$
\sigma_{W^{ \pm} Z, \mathrm{NLO} \text { QCD+EW }}^{\text {tot. }}=45.8 \pm 0.7(\mathrm{PDF})+2.2 /-1.8(\mathrm{scale}) \mathrm{pb},
$$

where we have used $\operatorname{Br}\left(W \rightarrow e v_{e}\right)=10.86 \%$ and $\operatorname{Br}\left(Z \rightarrow \mu^{+} \mu^{-}\right)=3.3658 \%$ as provided in Ref. [35] to unfold the cross section as done in Ref. [1]. This result is to be compared with

$\sigma_{W^{ \pm} Z \text {,ATLAS }}^{\text {tot. }}=51.0 \pm 2.4 \mathrm{pb}$ as reported in Ref. [1], showing a good agreement at the $1.6 \sigma$ level. The agreement becomes even much better when the next-to-next-to-leading order QCD corrections, of the order of a $+11 \%$ on top of the NLO QCD results at $13 \mathrm{TeV}$ for our scale choice, are taken into account [11]. We note that the EW corrections to the total cross section are completely negligible (at the sub-permil level) because of the cancellation between the negative corrections to the $\bar{q} q^{\prime}$ channels and the positive corrections to the $q \gamma$ channels, in agreement with the finding in Ref. [8].

\section{III.1. Angular distributions and polarization fractions}

We first present here results for the $\cos \theta$ distributions, from which the polarization fractions are calculated. They are shown in Fig. 2, where the LO, NLO QCD, and NLO QCD+EW distributions are separately provided. The bands indicate the total theoretical uncertainty calculated as a linear sum of PDF and scale uncertainties at NLO QCD. The $K$ factor defined as

$$
K_{\mathrm{NLOQCD}}=\frac{d \sigma_{\mathrm{NLOQCD}}}{d \sigma_{\mathrm{LO}}}
$$



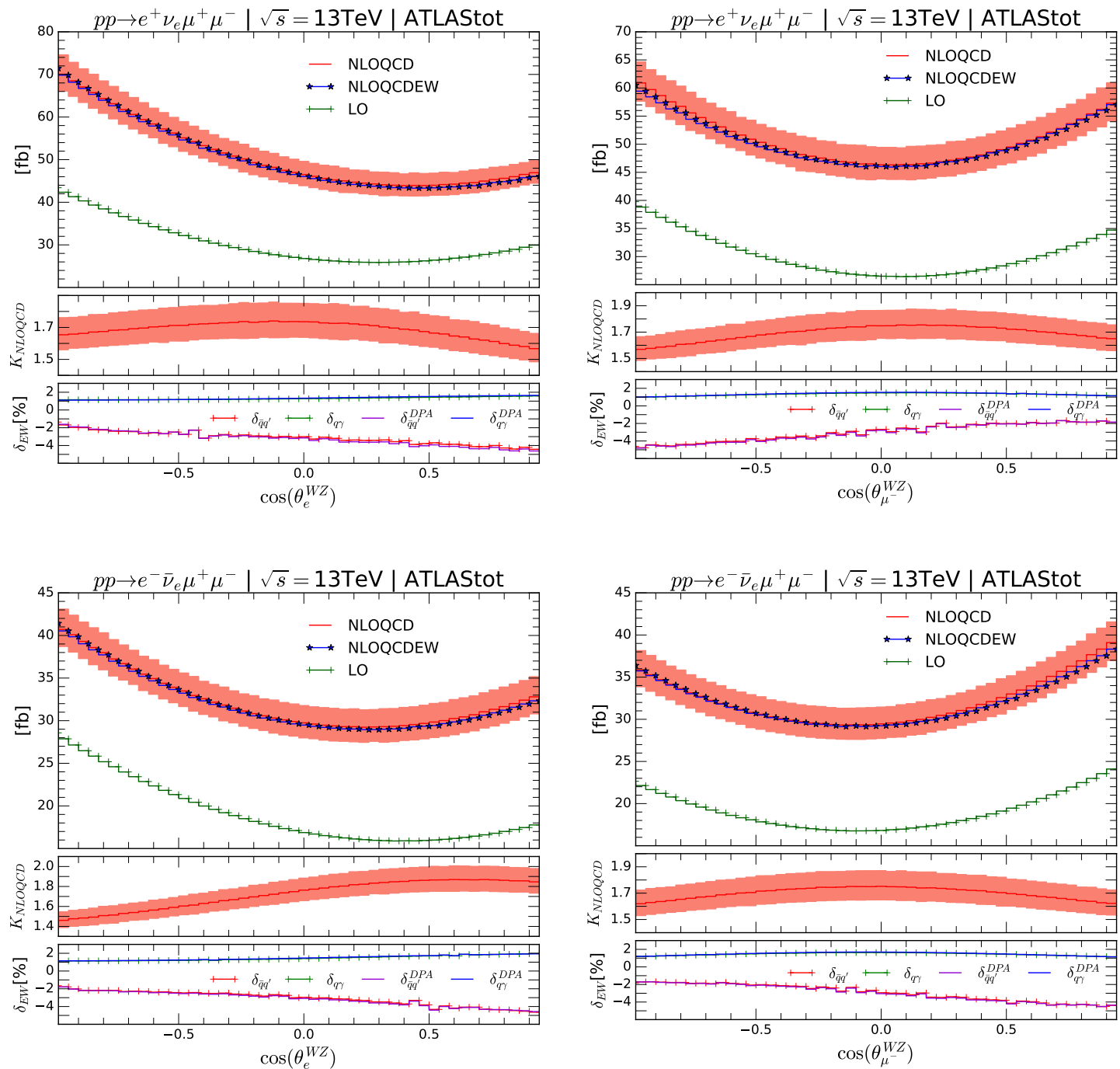

Fig. 2. Distributions of the $\cos \theta$ distributions of the (anti)electron (left column) and the muon (left column) for the process $W^{+} Z$ (top row) and $W^{-} Z$ (bottom row). The upper panels show the absolute values of the cross sections at LO (in green), NLO QCD (red), and NLO QCD+EW (blue). The middle panels display the ratio of the NLO QCD cross sections to the corresponding LO ones. The bands indicate the total theoretical uncertainty calculated as a linear sum of PDF and scale uncertainties at NLO QCD. The bottom panels show the NLO EW corrections (see text) calculated using DPA relative to the LO (marked with plus signs) and DPA LO cross sections.

is shown in the middle panels together with the corresponding uncertainty bands. To quantify the EW corrections, we define, as in Ref. [5], the following EW corrections

$$
\delta_{\bar{q} q^{\prime}}=\frac{d \Delta \sigma_{\bar{q} q^{\prime}}^{\mathrm{NLEW}}}{d \sigma^{\mathrm{LO}}}, \quad \delta_{q \gamma}=\frac{d \Delta \sigma_{q \gamma}^{\mathrm{NLOEW}}}{d \sigma^{\mathrm{LO}}},
$$


where the EW corrections to the quark anti-quark annihilation processes and to the photon quark induced processes are separated. The reason to show these corrections separately is to see to what extent they cancel each other. For the case of on-shell $W Z$ production, it has been shown in Ref. [8] that this cancellation is large. In this work, since leptonic decays are included, the QED final state photon radiation shifts the position of the di-muon invariant mass, leading to a shift in the photonradiated contribution to the $\delta_{\bar{q} q^{\prime}}$ correction. This shift is negative, making the $\delta_{\bar{q} q^{\prime}}$ correction more negative. As a result, we see that the total EW correction $\delta_{\mathrm{EW}}=\delta_{\bar{q} q^{\prime}}+\delta_{q \gamma}$ is negative, while it is more positive in Ref. [8]. Another important difference between this work and Ref. [8] is that different photon PDFs are used. This also changes the $q \gamma$ contribution significantly.

In order to see the effects of the DPA approximation at LO, we replace the denominators in Eq. (10) by the DPA LO results. This gives

$$
\delta_{\bar{q} q^{\prime}}^{\mathrm{DPA}}=\frac{d \Delta \sigma_{\bar{q} q^{\prime}}^{\mathrm{NLOEW}}}{d \sigma_{\mathrm{DPA}}^{\mathrm{LO}}}, \quad \delta_{q \gamma}^{\mathrm{DPA}}=\frac{d \Delta \sigma_{q \gamma}^{\mathrm{NLOEW}}}{d \sigma_{\mathrm{DPA}}^{\mathrm{LO}}},
$$

which are also shown in the bottom panels in Fig. 2 for the sake of comparison. The EW corrections $\delta_{\mathrm{EW}}$ are the same when compared to DPA LO or LO, while in Ref. [5] there are some differences especially at large negative $\cos \theta$ values. The effect of inclusive cuts is thus here visible.

We see that the NLO QCD corrections are large, varying in the range from $40 \%$ to $100 \%$ compared to the LO cross section, while the NLO EW corrections are very small in magnitude, as already known [8]. However it is important to note that the shape of the angular distributions is different between the EW corrections and the QCD corrections, a new feature which has an impact on the polarization fractions. We see clearly that the QCD corrections are not constant, but the shape distortion effect is not that large except in the $\cos \theta_{e^{-}}$in the $W^{-} Z$ channel where the QCD $K$-factor starts at $K_{N L O Q C D} \simeq 1.5$ for large negative $\cos \theta$ values and reaches $K_{N L O Q C D} \simeq 1.85$ at large positive values. The EW corrections also introduce some visible shape distortion effects. Comparisons between the $W^{+} Z$ and $W^{-} Z$ channels are valuable as charge asymmetry observables can be measured. In this context, it is interesting to notice that the QCD corrections are very similar in the $\cos \theta_{\mu^{-}}$distributions, but very different in the $\cos \theta_{e}$ distributions. Remarkably, the opposite behaviors are observed in the EW corrections, for both $\bar{q} q^{\prime}$ and $q \gamma$ corrections. The large charge asymmetry in the QCD corrections to the $\cos \theta_{e}$ distribution is most probably due to the $q g$ induced processes which first occur at NLO. On the other hand, the large effect observed in the EW corrections to the $\cos \theta_{\mu^{-}}$distribution is due to the QED final state radiation. This means that the charge asymmetries in $W$ polarization fractions are more sensitive to the gluon PDF than in the $Z$ case.

From the above $\cos \theta$ distributions, the polarization fractions are calculated. This result is presented in Table 1, where PDF and scale uncertainties associated with the LO and NLO QCD predictions are also calculated. To quantify the aforementioned higher-order effects on charge asymmetry observables, we define here two observables,

$$
\mathscr{A}_{L R}^{V}=\frac{q_{W^{+} Z}^{V}-q_{W^{-} Z}^{V}}{q_{W^{+} Z}^{V}+q_{W^{-} Z}^{V}}, \quad \mathscr{B}_{0}^{V}=\frac{p_{W^{+} Z}^{V}-p_{W^{-} Z}^{V}}{p_{W^{+} Z}^{V}+p_{W^{-} Z}^{V}},
$$

where $q^{V}=\left|f_{L}^{V}-f_{R}^{V}\right|$ and $p^{V}=\left|f_{0}^{V}\right|$. Note that, absolute values are needed because, in general, the fractions can get negative as shown in Ref. [5] for the case of the fiducial distributions in the 
Table 1. $W$ and $Z$ polarization fractions in the processes $p p \rightarrow e^{+} v_{e} \mu^{+} \mu^{-}$(upper rows) and $p p \rightarrow e^{-} v_{e} \mu^{+} \mu^{-}$(lower rows) at DPA LO, LO, NLO EW, NLO QCD, and NLO $\mathrm{QCD}+\mathrm{EW}$. The PDF uncertainties (in parenthesis) and the scale uncertainties are provided for the LO and NLO QCD results, all given on the last digit of the central prediction.

\begin{tabular}{|c|c|c|c||c|c|c|}
\hline Method & $f_{L}^{W}$ & $f_{0}^{W}$ & $f_{R}^{W}$ & $f_{L}^{Z}$ & $f_{0}^{Z}$ & $f_{R}^{Z}$ \\
\hline $\mathrm{DPALO}\left(W^{+} Z\right)$ & 0.515 & 0.153 & 0.332 & 0.333 & 0.144 & 0.522 \\
\hline $\mathrm{LO}\left(W^{+} Z\right)$ & $0.482(1)_{-1}^{+1}$ & $0.181(1)_{-2}^{+1}$ & $0.337(0.2)_{-0.5}^{+1}$ & $0.306(1)_{-1}^{+1}$ & $0.164(0.4)_{-1}^{+1}$ & $0.529(0.3)_{-0.2}^{+0.3}$ \\
\hline $\mathrm{NLOEW}\left(W^{+} Z\right)$ & 0.486 & 0.180 & 0.334 & 0.335 & 0.169 & 0.496 \\
\hline $\mathrm{NLOQCD}\left(W^{+} Z\right)$ & $0.471(1)_{-1}^{+1}$ & $0.218(1)_{-3}^{+3}$ & $0.311(1)_{-2}^{+2}$ & $0.338(1)_{-3}^{+3}$ & $0.209(1)_{-3}^{+4}$ & $0.453(1)_{-6}^{+6}$ \\
\hline $\mathrm{NLOQCDEW}\left(W^{+} Z\right)$ & 0.473 & 0.218 & 0.309 & 0.355 & 0.212 & 0.433 \\
\hline \hline $\mathrm{DPALO}\left(W^{-} Z\right)$ & 0.329 & 0.158 & 0.513 & 0.520 & 0.150 & 0.331 \\
\hline $\mathrm{LO}\left(W^{-} Z\right)$ & $0.316(0.4)_{-1}^{+1}$ & $0.181(0.4)_{-1}^{+1}$ & $0.503(0.3)_{-1}^{+1}$ & $0.501(1)_{-0.4}^{+1}$ & $0.168(0.4)_{-1}^{+1}$ & $0.332(0.4)_{-1}^{+1}$ \\
\hline $\mathrm{NLOEW}\left(W^{-} Z\right)$ & 0.313 & 0.181 & 0.506 & 0.470 & 0.172 & 0.358 \\
\hline $\mathrm{NLOQCD}\left(W^{-} Z\right)$ & $0.344(1)_{-2}^{+3}$ & $0.225(1)_{-3}^{+3}$ & $0.431(1)_{-6}^{+6}$ & $0.478(1)_{-2}^{+1}$ & $0.208(1)_{-3}^{+3}$ & $0.314(1)_{-2}^{+2}$ \\
\hline $\mathrm{NLOQCDEW}\left(W^{-} Z\right)$ & 0.342 & 0.226 & 0.432 & 0.459 & 0.211 & 0.329 \\
\hline
\end{tabular}

Table 2. Left-right charge asymmetries.

\begin{tabular}{|c|c|c|c|c|c|}
\hline Asymmetry & DPALO & LO & NLOEW & NLOQCD & NLOQCDEW \\
\hline $\mathscr{A}_{L R}^{W}$ & $-27.0 \%$ & $-12.7 \%$ & $-11.9 \%$ & $+29.6 \%$ & $+29.1 \%$ \\
\hline $\mathscr{A}_{L R}^{Z}$ & $0 \%$ & $+13.8 \%$ & $+17.9 \%$ & $-17.6 \%$ & $-25.0 \%$ \\
\hline
\end{tabular}

Collins-Soper coordinate system. Furthermore, since the three fractions sum to unity, only two parameters are independent. From Table 1 we see that $\mathscr{B}_{0}^{V}$ is not interesting as this asymmetry is very small, namely $\mathscr{B}_{0}^{W} \approx-2 \%$ and $\mathscr{B}_{0}^{Z} \approx+0.2 \%$ at NLO QCD+EW accuracy. However, the left-right charge asymmetries $\mathscr{A}_{L R}^{V}$ are much larger, being $\mathscr{A}_{L R}^{W} \approx+29 \%$ and $\mathscr{A}_{L R}^{Z} \approx-25 \%$ at NLO QCD+EW accuracy. Results at DPA LO, LO, NLO EW and NLO QCD levels are provided in Table 2, showing that these observables are very sensitive to off-shell and higher-order effects as the left-right asymmetries are negligible in the DPA limit, being numerically at the per mill level for the $W$ case and even smaller for the $Z$ case. Consistently with the above observations on the distributions, we see that the $W$ asymmetry is more sensitive to the QCD corrections, while the $Z$ asymmetry is more sensitive to the EW corrections. Note that the theory uncertainties cancel in the ratios defining the left-right asymmetries and are then expected to be negligible. We will not discuss them further.

We close this section by presenting in Table 3 the NLO QCD+EW results of the fractions for different invariant mass windows of the muon pair. These cuts are named CUT-i, $i=1, \ldots, 6$, corresponding respectively to $(86 \mathrm{GeV}, 96 \mathrm{GeV}),(81 \mathrm{GeV}, 101 \mathrm{GeV}),(76 \mathrm{GeV}, 106 \mathrm{GeV}),(71$ $\mathrm{GeV}, 111 \mathrm{GeV}),(66 \mathrm{GeV}, 116 \mathrm{GeV})$, and $(60 \mathrm{GeV}, 120 \mathrm{GeV})$. Note that CUT-5 is the default cut 
Table 3. NLO QCD+EW $W$ and $Z$ polarization fractions at different cuts (see text).

\begin{tabular}{|c|c|c|c||c|c|c|}
\hline Cut & $f_{L}^{W}$ & $f_{0}^{W}$ & $f_{R}^{W}$ & $f_{L}^{Z}$ & $f_{0}^{Z}$ & $f_{R}^{Z}$ \\
\hline CUT-1 $\left(W^{+} Z\right)$ & 0.475 & 0.218 & 0.307 & 0.360 & 0.217 & 0.424 \\
CUT-2 $\left(W^{+} Z\right)$ & 0.475 & 0.217 & 0.308 & 0.359 & 0.214 & 0.427 \\
CUT-3 $\left(W^{+} Z\right)$ & 0.475 & 0.217 & 0.308 & 0.357 & 0.213 & 0.429 \\
CUT-4 $\left(W^{+} Z\right)$ & 0.474 & 0.217 & 0.309 & 0.356 & 0.213 & 0.431 \\
CUT-5 $\left(W^{+} Z\right)$ & 0.473 & 0.218 & 0.309 & 0.355 & 0.212 & 0.433 \\
CUT-6 $\left(W^{+} Z\right)$ & 0.473 & 0.218 & 0.310 & 0.353 & 0.212 & 0.435 \\
\hline \hline CUT-1 $\left(W^{-} Z\right)$ & 0.341 & 0.227 & 0.432 & 0.455 & 0.215 & 0.329 \\
CUT-2 $\left(W^{-} Z\right)$ & 0.341 & 0.226 & 0.433 & 0.457 & 0.213 & 0.330 \\
CUT-3 $\left(W^{-} Z\right)$ & 0.341 & 0.226 & 0.433 & 0.458 & 0.212 & 0.330 \\
CUT-4 $\left(W^{-} Z\right)$ & 0.342 & 0.226 & 0.433 & 0.459 & 0.212 & 0.329 \\
CUT-5 $\left(W^{-} Z\right)$ & 0.342 & 0.226 & 0.432 & 0.459 & 0.211 & 0.329 \\
CUT-6 $\left(W^{-} Z\right)$ & 0.343 & 0.226 & 0.432 & 0.460 & 0.211 & 0.329 \\
\hline
\end{tabular}

defined in Eq. (7), while CUT-6 is used by CMS [36] to define their total phase space. As expected, we see that the $W$ fractions are almost unchanged while the $Z$ fractions vary more visibly. For the $Z$ fractions, the different behaviors between the $W^{+} Z$ and $W^{-} Z$ channels are interesting. While the $f_{R}^{Z}$ in the former case varies most strongly, it is almost unchanged in the latter. This unexpected constant behavior is due to the opposite behaviors in the other fractions.

\section{III.2. Angular coefficients}

We now turn to the angular coefficients. Results for the (anti)electron are presented in Table 4 and for the muon in Table 5 at LO, NLO QCD, NLO EW, and NLO QCD+EW levels.

The first thing to notice is that the values of the P-odd coefficients $A_{5-7}$ are very small, but non-vanishing. In order to see that they are indeed statistically non-zero, we show the DPA LO and LO results together with statistical errors in Table 6 for the (anti)electron case. For completeness, similar results for the muon case are also provided in Table 7. We see that those coefficients are zero within the statistical error in the DPA. However, at LO, when full off-shell effects are taken into account, they are all non-zero, except $A_{5}^{e^{+}}$and $A_{5, W^{+} Z}^{\mu^{-}}$where the results are very small. It has been shown in $[5,21]$ that, in the DPA, $A_{5-7}$ are proportional to the imaginary part of the spin-density matrices. This means that in the zero-width limit (i.e. $\Gamma_{V} \rightarrow 0$ ) they are vanishing, explaining why they are so small in the DPA. Note that, a finite width induces an off-shell effect, but the full off-shell effects at LO include additionally new Feynman diagrams such as virtual photon and single-resonant contributions. This explains why the values of the P-odd coefficients are significantly larger at LO than at DPA LO. Results in Table 4 and Table 5 show that they remain very small at NLO QCD+EW accuracy. Measuring them in experiments is therefore very challenging (see [20] for a study on $W$-jet production at the LHC). 
Table 4. Angular coefficients of the $e^{+}$(upper rows) and $e^{-}$(lower rows) distributions for the final states $e^{+} v_{e} \mu^{+} \mu^{-}$and $e^{-} \bar{v}_{e} \mu^{+} \mu^{-}$, respectively, at LO, NLO EW, NLO QCD, and NLO QCD+EW. The PDF uncertainties (in parenthesis) and the scale uncertainties are provided for the LO and NLO QCD results, all given on the last digit of the central prediction.

\begin{tabular}{|c|c|c|c|c|c|c|c|c|}
\hline Method & $A_{0}$ & $A_{1}$ & $A_{2}$ & $A_{3}$ & $A_{4}$ & $A_{5}$ & $A_{6}$ & $A_{7}$ \\
\hline $\mathrm{LO}\left(W^{+} Z\right)$ & $0.363(1)_{-3}^{+3}$ & $-0.049(1)_{-2}^{+1}$ & $-0.232(1)_{-4}^{+4}$ & $-0.102(2)_{-2}^{+1}$ & $-0.289(1)_{-1}^{+1}$ & $-0.0002(1)_{-1}^{+1}$ & $-0.003(0.3)_{-0.01}^{+0.1}$ & $-0.011(0.5)_{-0.03}^{+0.2}$ \\
\hline $\mathrm{NLOEW}\left(W^{+} Z\right)$ & 0.359 & -0.052 & -0.215 & -0.091 & -0.304 & -0.001 & -0.005 & -0.007 \\
\hline $\mathrm{NLOQCD}\left(W^{+} Z\right)$ & $0.436(1)_{-5}^{+6}$ & $-0.120(1)_{-6}^{+6}$ & $-0.233(1)_{-2}^{+3}$ & $-0.021(2)_{-7}^{+7}$ & $-0.319(2)_{-3}^{+3}$ & $0.0003(5)_{-0.4}^{+2}$ & $-0.001(1)_{-0.2}^{+0.01}$ & $-0.001(0.4)_{-1}^{+1}$ \\
\hline $\mathrm{NLOQCDEW}\left(W^{+} Z\right)$ & 0.435 & -0.122 & -0.222 & -0.014 & -0.328 & -0.0002 & -0.002 & 0.001 \\
\hline \hline $\mathrm{LO}\left(W^{-} Z\right)$ & $0.362(1)_{-3}^{+3}$ & $-0.067(1)_{-2}^{+2}$ & $-0.253(1)_{-3}^{+4}$ & $0.118(2)_{-3}^{+4}$ & $-0.374(1)_{-0.2}^{+0.2}$ & $0.002(0.2)_{-0.03}^{+0}$ & $0.002(0.4)_{-0.1}^{+0}$ & $0.007(0.4)_{-0.02}^{+0.2}$ \\
\hline $\mathrm{NLOEW}\left(W^{-} Z\right)$ & 0.362 & -0.072 & -0.232 & 0.127 & -0.388 & 0.003 & 0.004 & 0.0005 \\
\hline $\mathrm{NLOQCD}\left(W^{-} Z\right)$ & $0.451(1)_{-7}^{+7}$ & $-0.131(1)_{-5}^{+5}$ & $-0.233(2)_{-4}^{+4}$ & $0.032(2)_{-8}^{+7}$ & $-0.174(4)_{-16}^{+17}$ & $0.001(1)_{-0.1}^{+0.3}$ & $0.001(0.4)_{-0.1}^{+0.2}$ & $-0.005(0.3)_{-1}^{+1}$ \\
\hline $\mathrm{NLOQCDEW}\left(W^{-} Z\right)$ & 0.451 & -0.135 & -0.219 & 0.037 & -0.180 & 0.002 & 0.002 & -0.009 \\
\hline
\end{tabular}

Table 5. Same as Table 4 but for the $\mu^{-}$coefficients.

\begin{tabular}{|c|c|c|c|c|c|c|c|c|}
\hline Method & $A_{0}$ & $A_{1}$ & $A_{2}$ & $A_{3}$ & $A_{4}$ & $A_{5}$ & $A_{6}$ & $A_{7}$ \\
\hline $\mathrm{LO}\left(W^{+} Z\right)$ & $0.329(1)_{-3}^{+2}$ & $-0.034(1)_{-2}^{+2}$ & $-0.145(1)_{-2}^{+3}$ & $0.032(1)_{-1}^{+1}$ & $-0.096(0.3)_{-0.3}^{+0.4}$ & $0.000004(209)_{-83}^{+87}$ & $-0.011(0.2)_{-0.1}^{+0.1}$ & $0.013(0.2)_{-0.2}^{+0.05}$ \\
\hline $\mathrm{NLOEW}\left(W^{+} Z\right)$ & 0.338 & -0.038 & -0.136 & 0.041 & -0.069 & -0.0005 & -0.012 & 0.013 \\
\hline $\mathrm{NLOQCD}\left(W^{+} Z\right)$ & $0.418(1)_{-7}^{+7}$ & $-0.096(1)_{-6}^{+5}$ & $-0.144(1)_{-2}^{+2}$ & $0.010(1)_{-2}^{+2}$ & $-0.049(1)_{-4}^{+4}$ & $0.0002(10)_{-0.04}^{+2}$ & $-0.008(0.3)_{-0.2}^{+0.2}$ & $0.009(0.2)_{-0.3}^{+0.2}$ \\
\hline $\mathrm{NLOQCDEW}\left(W^{+} Z\right)$ & 0.424 & -0.100 & -0.139 & 0.015 & -0.033 & -0.0002 & -0.009 & 0.009 \\
\hline \hline $\mathrm{LO}\left(W^{-} Z\right)$ & $0.335(1)_{-3}^{+3}$ & $0.013(1)_{-2}^{+2}$ & $-0.153(1)_{-2}^{+2}$ & $0.012(0.4)_{-0.1}^{+0.2}$ & $0.072(0.3)_{-0.2}^{+0.2}$ & $0.004(0.2)_{-0.04}^{+0.1}$ & $0.009(0.2)_{-0.1}^{+0.1}$ & $0.014(0.2)_{-0.2}^{+0.1}$ \\
\hline $\mathrm{NLOEW}\left(W^{-} Z\right)$ & 0.344 & 0.008 & -0.146 & 0.014 & 0.048 & 0.004 & 0.011 & 0.014 \\
\hline $\mathrm{NLOQCD}\left(W^{-} Z\right)$ & $0.417(1)_{-6}^{+6}$ & $-0.071(1)_{-7}^{+7}$ & $-0.164(1)_{-1}^{+2}$ & $0.001(0.5)_{-1}^{+1}$ & $0.070(0.5)_{-0.3}^{+0.3}$ & $0.002(0.5)_{-0.1}^{+0.2}$ & $0.006(1)_{-0.2}^{+0.2}$ & $0.009(1)_{-0.3}^{+0.3}$ \\
\hline $\mathrm{NLOQCDEW}\left(W^{-} Z\right)$ & 0.423 & -0.075 & -0.160 & 0.002 & 0.056 & 0.003 & 0.007 & 0.009 \\
\hline
\end{tabular}

We now focus on the P-even coefficients $A_{1-4}$. Results at the NLO QCD+EW accuracy in Table 4 and Table 5 show that they are large, hence should be measureable. EW corrections are significant for $A_{3}^{\mu^{-}}$and $A_{4}^{\mu^{-}}$due to the radiative corrections to the $Z \rightarrow \mu^{+} \mu^{-}$decay, as found in Ref. [5] using more exclusive cuts.

Similarly to the previous section, we can define various charge asymmetries as

$$
\mathscr{C}_{i}^{V}=\frac{r_{i, W^{+} Z}^{V}-r_{i, W^{-} Z}^{V}}{r_{i, W^{+} Z}^{V}+r_{i, W^{-} Z}^{V}}, \quad r_{i}^{W}=\left|A_{i}^{e}\right|, \quad r_{i}^{Z}=\left|A_{i}^{\mu^{-}}\right|, i=1, \ldots, 7 .
$$


Table 6. Angular coefficients of the $e^{+}$(upper rows) and $e^{-}$(lower rows) distributions for the final states $e^{+} v_{e} \mu^{+} \mu^{-}$and $e^{-} \bar{v}_{e} \mu^{+} \mu^{-}$, respectively, at DPA LO and LO. The numbers in square brackets represent the statistical error, when it is significant.

\begin{tabular}{|c|c|c|c|c|c|c|c|c|}
\hline Method & $A_{0}$ & $A_{1}$ & $A_{2}$ & $A_{3}$ & $A_{4}$ & $A_{5}$ & $A_{6}$ & $A_{7}$ \\
\hline $\operatorname{DPALO}\left(W^{+} Z\right)$ & 0.306 & -0.030 & -0.286 & 0.092 & -0.368 & $-0.00001[40]$ & $-0.0001[2]$ & $0.00005[19]$ \\
\hline $\mathrm{LO}\left(W^{+} Z\right)$ & 0.363 & -0.049 & -0.232 & -0.102 & -0.289 & $-0.0002[5]$ & $-0.0034[4]$ & -0.011 \\
\hline \hline $\operatorname{DPALO}\left(W^{-} Z\right)$ & 0.317 & $-0.0023[2]$ & -0.295 & -0.082 & -0.367 & $0.00001[38]$ & $-0.0001[2]$ & $0.0001[2]$ \\
\hline $\mathrm{LO}\left(W^{-} Z\right)$ & 0.362 & -0.066 & -0.254 & 0.118 & -0.374 & $0.0024[4]$ & $0.0024[3]$ & $0.0067[2]$ \\
\hline
\end{tabular}

Table 7. Same as Table 6 but for the $\mu^{-}$distributions.

\begin{tabular}{|c|c|c|c|c|c|c|c|c|}
\hline Method & $A_{0}$ & $A_{1}$ & $A_{2}$ & $A_{3}$ & $A_{4}$ & $A_{5}$ & $A_{6}$ & $A_{7}$ \\
\hline $\operatorname{DPALO}\left(W^{+} Z\right)$ & 0.289 & 0.037 & -0.183 & -0.026 & -0.081 & $-0.0001[2]$ & $-0.0001[1]$ & $0.00001[17]$ \\
\hline $\mathrm{LO}\left(W^{+} Z\right)$ & 0.329 & -0.034 & -0.145 & 0.032 & -0.096 & $0.0001[4]$ & -0.011 & 0.013 \\
\hline \hline $\operatorname{DPALO}\left(W^{-} Z\right)$ & 0.299 & 0.026 & -0.188 & $-0.0085[1]$ & 0.081 & $-0.00003[19]$ & $-0.0001[2]$ & $0.00002[14]$ \\
\hline $\mathrm{LO}\left(W^{-} Z\right)$ & 0.336 & 0.013 & -0.154 & 0.012 & 0.072 & $0.0041[8]$ & $0.0091[4]$ & 0.014 \\
\hline
\end{tabular}

Because of the relations between $f_{L, R, 0}$ and $A_{0,4}$ presented in Eq.(4), the asymmetries $\mathscr{C}_{0}^{V}$ and $\mathscr{C}_{4}^{V}$ are identical to $\mathscr{B}_{0}^{V}$ and $\mathscr{A}_{L R}^{V}$, respectively. Looking at Table 4 and Table 5 we see that $\mathscr{C}_{3}^{V}$ and $\mathscr{C}_{4}^{V}$ are the most interesting. Note that we ignore $\mathscr{C}_{5-7}^{V}$ in this discussion because they are very difficult to measure. Similar to Table 2, values for $\mathscr{C}_{3}^{V}$ in different approximations are provided in Table 8 . Compared to $\mathscr{A}_{L R}^{V}$, this asymmetry is much larger. Another interesting difference is the value at DPA LO. While it is almost vanishing for $\mathscr{A}_{L R}^{V}$, it is large for $\mathscr{C}_{3}^{V}$, reaching $+51 \%$ for the $Z$ case, suggesting very different origins. This is indeed the case because $A_{3}$ is related to the off-diagonal entries of the spin-density matrices at DPA LO, while $A_{4}$ comes from the diagonal ones [5]. This is also why the value of $A_{3}$ is much smaller than that of $A_{4}$. All in all, we see that the value of $\mathscr{C}_{3}^{V}$ is much larger hence could be an additional probe of the spin structure of the gauge bosons, but this observable is more difficult to measure than $\mathscr{A}_{L R}^{V}$ because of difficulties in measuring $A_{3}$.

Table 8. Charge asymmetries in the coefficient $A_{3}$.

\begin{tabular}{|c|c|c|c|c|c|}
\hline Asymmetry & DPALO & LO & NLOEW & NLOQCD & NLOQCDEW \\
\hline $\mathscr{C}_{3}^{W}$ & +0.057 & -0.073 & -0.165 & -0.208 & -0.451 \\
\hline $\mathscr{C}_{3}^{Z}$ & +0.507 & +0.455 & +0.491 & +0.818 & +0.765 \\
\hline
\end{tabular}




\section{CONCLUSIONS}

In this paper, we have presented results for polarization fractions and angular coefficients up to the NLO QCD+EW accuracy in the process $p p \rightarrow e^{ \pm} v_{e} \mu^{+} \mu^{-}$at the $13 \mathrm{TeV}$ LHC. We have used by default the total phase space adopted by ATLAS in their latest $13 \mathrm{TeV}$ analysis of the $W$ and $Z$ polarization observables, measured in the modified helicity coordinate system where the $z$-axis is defined as the gauge boson flight direction in the $W Z$ center-of-mass frame. Our LO and NLO QCD results include full off-shell effects while the EW corrections have been calculated in the double-pole approximation.

We have defined left-right charge asymmetries $\mathscr{A}_{L R}^{W / Z}$ out of the polarization fractions that are very sensitive to higher-order effects, in particular $\mathscr{A}_{L R}^{W}$ is sensitive to the QCD corrections while $\mathscr{A}_{L R}^{Z}$ is sensitive to the EW corrections. This sensitivity can be related to the shape of the higher-order corrections to the angular distributions underlying the calculations of the polarization fractions. These asymmetries are found to be large at NLO QCD+EW order, of the order of $+29 \%$ for the $W$ boson and $-25 \%$ for the $Z$ boson, that should be measurable at the LHC.

We have also defined similar charge asymmetries for the angular coefficients themselves and we have found that the asymmetries related to the P-even coefficient $A_{3}$ can also be very large, of the order of $-45 \%$ for the $W$ boson and $+78 \%$ for the $Z$ boson, and could be additional probes of the polarization structure of the underlying dynamics, even if they are more difficult to measure than the left-right charge asymmetries. The EW corrections are also found to be sizable in these $A_{3}$ charge asymmetries.

This work is a step towards a study of the polarization observables that is as close as possible to the current experimental setup in ATLAS.

\section{ACKNOWLEDGMENT}

This work is funded by the Vietnam National Foundation for Science and Technology Development (NAFOSTED) under grant number 103.01-2017.78. We are grateful to Emmanuel Sauvan for fruitful discussions.

\section{REFERENCES}

[1] M. Aaboud et al., Eur. Phys. J. C79 (2019) 535.

[2] C. L. Bilchak, R. W. Brown and J. D. Stroughair, Phys. Rev. D29 (1984) 375-386.

[3] S. S. D. Willenbrock, Annals Phys. 186 (1988) 15.

[4] W. J. Stirling and E. Vryonidou, JHEP 07 (2012) 124.

[5] J. Baglio and L. D. Ninh, JHEP 04 (2019) 065.

[6] B. Biedermann, A. Denner and L. Hofer, JHEP 10 (2017) 043.

[7] A. Bierweiler, T. Kasprzik and J. H. Kühn, JHEP 12 (2013) 071.

[8] J. Baglio, L. D. Ninh and M. M. Weber, Phys. Rev. D88 (2013) 113005, [Erratum: Phys. Rev.D94,no.9,099902(2016)].

[9] J. Ohnemus, Phys. Rev. D44 (1991) 3477-3489.

[10] S. Frixione, P. Nason and G. Ridolfi, Nucl. Phys. B383 (1992) 3-44.

[11] M. Grazzini, S. Kallweit, D. Rathlev and M. Wiesemann, Phys. Lett. B761 (2016) 179-183.

[12] M. Grazzini, S. Kallweit, D. Rathlev and M. Wiesemann, JHEP 05 (2017) 139.

[13] J. C. Collins and D. E. Soper, Phys. Rev. D16 (1977) 2219.

[14] Z. Bern et al., Phys. Rev. D84 (2011) 034008.

[15] K. Arnold et al., Comput. Phys. Commun. 180 (2009) 1661-1670. 
[16] J. Baglio et al., arXiv:1404.3940 (2014) .

[17] M. Chaichian, M. Hayashi and K. Yamagishi, Phys. Rev. D25 (1982) 130, [Erratum: Phys. Rev.D26,2534(1982)].

[18] E. Mirkes, Nucl. Phys. B387 (1992) 3-85.

[19] K. Hagiwara, K.-i. Hikasa and N. Kai, Phys. Rev. Lett. 52 (1984) 1076.

[20] R. Frederix, K. Hagiwara, T. Yamada and H. Yokoya, Phys. Rev. Lett. 113 (2014) 152001.

[21] J. A. Aguilar-Saavedra and J. Bernabeu, Phys. Rev. D93 (2016) 011301.

[22] J. A. Aguilar-Saavedra, J. Bernabéu, V. A. Mitsou and A. Segarra, Eur. Phys. J. C77 (2017) 234.

[23] A. Manohar, P. Nason, G. P. Salam and G. Zanderighi, Phys. Rev. Lett. 117 (2016) 242002.

[24] A. V. Manohar, P. Nason, G. P. Salam and G. Zanderighi, JHEP 12 (2017) 046.

[25] J. Butterworth et al., J. Phys. G43 (2016) 023001.

[26] S. Dulat et al., Phys. Rev. D93 (2016) 033006.

[27] L. A. Harland-Lang, A. D. Martin, P. Motylinski and R. S. Thorne, Eur. Phys. J. C75 (2015) 204.

[28] R. D. Ball et al., JHEP 04 (2015) 040.

[29] J. Gao and P. Nadolsky, JHEP 07 (2014) 035.

[30] S. Carrazza, S. Forte, Z. Kassabov, J. I. Latorre and J. Rojo, Eur. Phys. J. C75 (2015) 369.

[31] G. Watt and R. S. Thorne, JHEP 08 (2012) 052.

[32] D. de Florian, G. F. R. Sborlini and G. Rodrigo, Eur. Phys. J. C76 (2016) 282.

[33] A. Buckley et al., Eur. Phys. J. C75 (2015) 132.

[34] M. Aaboud et al., Phys. Lett. B762 (2016) 1-22.

[35] K. Olive et al., Chin.Phys. C38 (2014) 090001.

[36] V. Khachatryan et al., Phys. Lett. B766 (2017) 268-290. 